\title{
BCOR-CCNB3 Sarcoma with Concurrent RNF213- SLC26A11 Gene Fusion: A Rare Sarcoma with Altered Histopathological Features After Chemotherapy
}

\author{
Wen Huang \\ The First Affiliated Hospital of USTC: Anhui Provincial Hospital \\ Wei Wang \\ The First Affiliated Hospital of USTC: Anhui Provincial Hospital \\ Liang-Liang Huang \\ The First Affiliated Hospital of USTC: Anhui Provincial Hospital \\ Heng Li \\ The First Affiliated Hospital of USTC: Anhui Provincial Hospital \\ Wen-Chao Zhou \\ The First Affiliated Hospital of USTC: Anhui Provincial Hospital \\ Hai-Bo Wu (D 406511983@qq.com) \\ The First Affiliated Hospital of USTC: Anhui Provincial Hospital
}

\section{Case report}

Keywords: BCOR-CCNB3, Gene fusion, Ewing-like sarcomas, RNF213-SLC26A11, Soft tissues

Posted Date: November 8th, 2021

DOI: https://doi.org/10.21203/rs.3.rs-1037017/v1

License: (c) (1) This work is licensed under a Creative Commons Attribution 4.0 International License.

Read Full License 


\section{Abstract}

Background Chemotherapy is a common approach for cancer treatment, but intrinsic genetic mutations in different individuals may cause different responses to chemotherapy, resulting in unique histopathological changes. The genetic mutation along with the distinct histopathological features may indicate new tumor entities. BCOR-CCNB3 sarcomas are a kind of Ewing-like sarcomas (ELS) occurring mostly in bone and soft tissues. No gene fusion other than BCOR-CCNB3 has been found in this type of tumor.

Case presentation Here, we report a case of 17-year-old male patient presented with a mass on his left shoulder that was diagnosed as undifferentiated small round cell sarcoma according to core biopsy. The patient received 5 courses of pre-operational chemotherapy, and the tumor was resected and analyzed. We observed primitive small round cells and larger myoid cells in the resected tumor tissue but not in biopsy, arterioles stenosis and occlusion were also detected, indicating a dramatic change of histopathological features of this tumor. In addition, the immunohistochemical results showed the altered staining patterns of BCOR, bcl2, CyclinD1, TLE1, AR, SMA, CD117, STAB2, CD56 and CD99 in tumor tissues after chemotherapy. Importantly, RNA sequencing revealed a RNF213-SLC26A11 fusion in the tumor sample.

Conclusions We propose that the BCOR-CCNB3 sarcoma with RNF213-SLC26A11 fusion may indicate a subset of tumors that undergo histopathological changes in response to chemotherapy. More similar cases in the future may help to clarify the clinical meanings of RNF213-SLC26A11 fusion in BCOR-CCNB3 sarcomas and the underlying mechanisms.

\section{Background}

Primitive small blue round cell tumors (SBRCTs) are malignant soft tissue sarcomas common in children and adolescents. Ewing sarcoma (ES) is the prototypical SBRCT characterized by the fusion of EWSR1 and a member of the ETS family of transcription factors. With the rapid development of molecular diagnostics, new entities of SBRCTs have been recognized. Recent reports described a subset of SBRCTs, named Ewing-like sarcomas (ELS), which are morphologically similar to ES. ELS contain a broad spectrum of tumors with different gene fusions, and $C / C$-rearranged sarcomas as the most common ELS account for about two-thirds of ELS [1]. In 2012, Pierron et al. reported BCOR-CCNB3 sarcomas as a kind of ELS that often occur in bone and soft tissues [2]. Morphologically, BCOR-CCNB3 sarcomas are highly cellular sarcomas that are composed of varying spindle and ovoid cells, monomorphic nuclei angulated with finely chromatin and indistinct nucleoli, and prominent delicate capillary network. The stroma showed varying amounts of myxoid and collagen $[3,4]$, and the morphologic spectrum of BCOR-CCNB3 sarcomas was quite varied. Increased cell density and obvious pleomorphism was observed in the recurrent and metastatic lesions of $B C O R-C C N B 3$ sarcomas [3-5]. In addition, heterogeneity in morphology appeared in BCOR-CCNB3 sarcomas after chemotherapy, along with possible changes of immunophenotypes such as decreased or loss of expression of CCNB3 and SATB2 [6-8]. 
The BCOR-CCNB3 sarcomas may have genetic changes such as HOX family and NTRK3 up-regulation [4], but no gene fusions other than the BCOR-CCNB3 fusion has been reported so far. The RNF213-SLC26A11 gene fusion has been only found in chronic myeloid leukemia[9] and glioma [10]. Here, we describe a BCOR-CCNB3 sarcoma with concurrent RNF213-SLC26A11 gene fusion showing unique morphologic features and peculiar immunophenotype after chemotherapy.

\section{Case Presentation}

\section{Case description}

A 17-year-old male patient presented with a mass on his left shoulder without apparent cause 6 months ago. This patient received no treatment until the mass enlarged with pain and discomfort. MRI showed a huge soft tissue mass $(10.0 \mathrm{~cm} \times 8.1 \mathrm{~cm} \times 6.6 \mathrm{~cm})$ growing around the left scapula with the imaging features of long T1, long T2, and high DWI signal (Fig. 1A). The tumor showed invasive growth and caused irregular left scapula destruction, extending into left supraspinatus, infraspinatus, subscapularis, spina scapulae of deltoid and other soft tissues. Contrast-enhanced CT showed unevenly mild to moderate enhancement of the mass which grew inside and outside the shoulder blade, and even above and below the scapular spine (Fig. 1B). The scapula presented sieve-like bone destruction, while no obvious bone destruction was found in the adjacent ribs, clavicle and humeral head. Pathological results of core biopsy suggested an undifferentiated small round cell sarcoma without genetic test. The patient received five courses of neoadjuvant chemotherapy (with the regimen of oxaliplatin, pirarubicin and ifosfamide). After then, he received radical resection of left shoulder blade followed by three circles of ifosfamide chemotherapy. No recurrence and metastasis developed half a year after surgery.

\section{Immunohistochemical Stains}

The 3- $\mu \mathrm{m}$-thick formalin-fixed paraffin-embedded (FFPE) slides were continuously sectioned for immunohistochemical staining of BCOR, Bcl2, CyclinD1, TLE1, AR, Ki67, SMA, STAB2, CD117, CD56 and CD99 with the Roche BenchMark ULTRA fully automated immunohistochemistry stainer.

\section{Fluorescence In Situ Hybridization (Fish)}

FISH for BCOR was performed on 4- $\mu$ m-thick FFPE sections with the Dual Color Probe according to the manufacturer's instruction (Guangzhou LBP Medicine Science \& Technology Co.,Ltd., China). Two hundred non-overlapping intact nuclei were examined in each slide under a Leica fluorescence microscope. A specimen was considered positive when at least $20 \%$ of the nuclei showed a break-apart signal.

\section{Rna Sequencing}


Total RNA was extracted from FFPE samples using the RNeasy FFPE Kit (QIAGEN). RNA samples were quantified with BioAnalyzer 2100 (Agilent Technologies), depleted of ribosomal RNA (rRNA) and residual genomic DNA, purified with Agencourt RNA Clean XP Beads, and subjected to construction of the sequencing library with the KAPA Stranded RNA-Seq Library Preparation Kit. The resultant library was sequenced on Illumina HiSeq X Ten platform (Illumina) for paired-end 150bp sequencing. The outcome reads in FASTQ format were generated with bcl2fastq v2.16.0.10 software (Illumina), and the somatic fusion genes were identified using FusionCatcher pipeline with default settings (http://code.google.com/p/fusioncatcher/) [11]. The GRCh37/hg19 build was used as the human genome reference.

\section{Reverse Transcription Polymerase Chain Reaction (Rt-pcr)}

Reverse transcription polymerase chain reaction (RT-PCR)

RT-PCR was performed using the primers BCOR-E15-F: 5'-TCACGAACGAAATTCAGACTC-3' and CCNB3-E5R: 5'- GCTACTACTGGTGTGACTTCC-3' for detecting BCOR-CCNB3 fusion, and RNF213-E2-F: 5'AGGAGGAAACCCCCAAGTTC-3' and SLC26A11-E8-R: 5'-TCGAAGGAGTACGCAACCAG-3' for detecting RNF213-SLC26A11 fusion. For Sanger sequencing, BCOR-E15-F and RNF213-E2-F were used.

\section{Results}

\section{Altered histopathological features of the tumor after neoadjuvant chemotherapy}

In the initial core biopsy specimen collected before chemotherapy, the tumor cells were arranged in solid sheets, with the cell morphology of round or oval, eosinophilic cytoplasm, fine chromatin, indistinct nucleoli and rich capillary network (Fig. 1C). After neoadjuvant chemotherapy, the resected specimen showed infiltration and destruction of bone and muscle. Notably, the morphology and structure of the resected specimen were completely different from those of core biopsy. The tumor cells had a biphasic appearance (Fig. 1D) indicating primitive small round cells (usually distributed in small clusters) with scant cytoplasm, round nucleus, fine chromatin and inconspicuous nucleolus, and larger myoid cells with abundant eosinophilic cytoplasm, oval nucleus, rough chromatin and obvious small nucleolus. A local transition between the two types of cells was observed. The tumor cells were arrayed in various structures, such as solid, nested, trabecular and cords-like. Some crescent-shaped gaps appeared around the nests and formed a glomerular-like structure (Fig. 1E). The stroma showed varying degrees of edematous or hyaline degeneration, focal hemorrhage and small focal necrosis of tumor cells and scattered mast cell infiltration. Besides capillary, arterioles with obvious thickening walls, varying degrees of hyaline degeneration, vascular stenosis and occlusion were also detected (Fig. 1F).

The immunohistochemical staining showed the diffusely and strongly expression of $\mathrm{BCOR}, \mathrm{bcl} 2$, CyclinD1, TLE1 and AR in the biopsy specimen, but these markers were focal positive mainly in myoid 
cells in the radical specimen (Fig. 1G-1L). For SMA (Fig. 1M and 1N) and CD117, the staining was negative in the biopsy but positive in the resected tumor, mainly in myoid cells. STAB2, CD56 and CD99 were scattered positive in few cells in the biopsy tissue but focal positive in myoid cells in the resected tumor. The proliferation index of Ki67 decreased from 30-10\% after neoadjuvant chemotherapy. The immunohistochemistry changes in tumor core biopsy and radical specimen were summarized in Supplementary Table 1.

\section{RNA sequencing revealed the RNF213-SLC26A11 fusion in addition to the BCOR-CCNB3 gene fusion}

RNA sequencing identified a $B C O R-C C N B 3$ gene fusion in this case. This fusion joined the exons 1-15 of BCOR (ENST00000342274) to the exons 5-12 of CCNB3 (ENST00000276014) (Fig. 2A). In the tumor relative to normal control tissues, the reads of the BCOR exons showed a sharp decline at the $3^{\prime}$ end (average: 1000, 3' end: 100), and almost no read was observed in the first four exons of CCNB3. To validate the RNA sequencing results, primers covering BCOR exon 15 (BCOR-E15-F) and CCNB3 exon 5 (CCNB3-E5-R) were designed and RT-PCR was performed to obtain the fusion fragment. Sanger sequencing showed that the fusion joined the regular acceptor site of $C C N B 3$ exon 5 to the putative GGTGAG donor splice-site (just before the stop codon TGA) of $B C O R$, generating a BCOR-CCNB3 fusion protein. The break-down of the $B C O R$ gene at the genomic level was validated by FISH (Fig. 2B). Whereas no other gene fusion has been detected for BCOR-CCNB3 sarcoma so far, we discovered a novel RNF213$S L C 26 A 11$ fusion in this case. In detail, the RNF213 (ENST00000582970) exons 1-2 were joined to the SLC26A11 (ENST00000411502) exons 8-18, generating an in-frame RNF213-SLC26A11 isoform (Fig. 2C). RT-PCR and the subsequent Sanger sequencing proved the existence of this novel fusion gene.

\section{Discussion And Conclusions}

The $B C O R$ gene located at Xp11.4 encoded the ubiquitously expressed BCOR protein that interacts with BCL6 to enhance BCL6-mediated transcriptional repression [2, 4, 12]. Physiologically, BCOR plays an important role in pluripotency by regulating differentiation and cell fate determination [2]. In bone and soft tissue sarcomas, $B C O R$ gene mutated mainly through internal tandem duplications (ITD) and gene fusion, among which the BCOR-CCNB3 is the most common fusion. CCNB3 is a member of the cyclin $\mathrm{B}$ family and is mainly expressed in the germ cells of testis [4]. In addition to CCNB3, BCOR has been reported to be fused with $Z C 3 H 7 B, C I I T A, M A M L 3$ and $K M T 2 D$ [13]. However, no fusion gene other than $B C O R-C C N B 3$ has been reported in BCOR-CCNB3 sarcoma so far. The BCOR-CCNB3 sarcoma with RNF213-SLC26A11 gene fusion is the first discovered case, which is featured by the changes in morphology and immunophenotype after neoadjuvant chemotherapy.

The $B C O R-C C N B 3$ sarcoma is more common in men, mainly in older children and adolescents, more in bone than in soft tissue [4-6]. The core biopsy of the BCOR-CCNB3 sarcoma with RNF213-SLC26A11 gene fusion is similar to regular BCOR-CCNB3 sarcomas in terms of histology. Regular BCOR-CCNB3 sarcoma is often composed of round or oval cells in different proportions, distributing in solid sheets, with less cytoplasm, fine chromatin, indistinct nucleoli, and rich capillary network [4]. Other morphological 
features in this tumor include myxoid spindle cell component, solid areas of high cellularity alternating with less cellular areas, hemangiopericytoma-like pattern, whorls-like arrangement, trabeculae or cord formation, heterologous cartilaginous differentiation, and fascicular architecture reminiscent of infantile fibrosarcoma [6, 7, 14-17]. BCOR-CCNB3 sarcomas treated with neoadjuvant chemotherapy are usually similar to the untreated samples in morphology, but they may display hypocellular loose fibrous tissue, a highly myxoid and sclerotic stroma, coagulative tumor necrosis and elongated bland-appearing nuclei without mitotic activity. Sometimes there are spindle cells with slit-like spaces and extravasated erythrocytes, foci of extramedullary haematopoiesis, pleomorphic tumor cells with multi-nucleated cells. Epithelioid cells appear in small clusters or cords, with focally prominent nucleoli $[1,5-8,18]$. However, we observed previously undescribed morphological changes in the BCOR-CCNB3 sarcoma with RNF213SLC26A11 gene fusion after neoadjuvant chemotherapy. Most noticeably, two new types of cells, the small round cells similar to classic ES and the larger myoid cells resembling smooth muscle cells or myoepithelioma cells, present in the resected tumor tissue but not the biopsy. The myoid cells showed SMA staining, indicating myogenic differentiation. In addition, we observed a large number of arterioles with thickened or even occluded walls in this tumor, which has never been reported in BCOR-CCNB3 sarcomas. Our discovery extends the morphological spectrum of $B C O R-C C N B 3$ sarcomas after chemotherapy.

So far,there has been no commonly accepted immunohistochemical markers for BCOR-CCNB3 sarcomas. The diffuse positive expression of CCNB3 can help diagnosis, but the focal or weakly positive staining of CCNB3 occurs in solitary fibrous tumor (SFT), rhabdomyosarcoma, ES and fibrosarcoma [6]. Likewise, although BCOR is expressed in almost all the BCOR-CCNB3 sarcomas [6], its specificity is doubtful. In addition to sarcoma with BCOR ITDs, BCOR also expresses in SFT, synovial sarcoma, ES, malignant lymphoma and small cell carcinoma [6]. Even with the co-staining of CCNB3 and BCOR, the diagnosis could not exclude the possibility of SFT and ES. Androgen (AR) expression has been detected in various benign and malignant soft tissue tumors, including juvenile nasopharyngeal angiofibroma [19], undifferentiated pleomorphic sarcoma, fibrosarcoma, leiomyosarcoma, rhabdomyosarcoma, myxoid liposarcoma, and angiosarcoma [20]. We reported for the first time that AR is expressed in BCOR-CCNB3 sarcomas. The expression of AR in sarcoma often indicates potential progression [20], but it may also be a therapeutic target. The immunohistochemical phenotypes of BCOR-CCNB3 sarcomas often change after neoadjuvant chemotherapy. For example, CCNB3 and SATB2 often have weakened expression or no expression $[6-8,15,18]$. In this case, the expression of BCOR, bcl2, CyclinD1, TLE1, AR and Ki67 all decreased after chemotherapy. Interestingly, these markers were preserved in myoid cells completely or partially. Meanwhile, while SMA, SATB1 and CD117 expression were detected in resected tumor tissues but not in biopsy specimens, the restricted expression of SMA and SATB1 in myoid cells indicated the differentiation of the tumor in response to chemotherapy. However, so far there is no clue for the origin of the small round cells.

The RNF213 gene locates on chromosome 17q25.3, coding for a ring finger protein with ubiquitin ligase and ATPase activities, which plays an important role in angiogenesis and inflammatory responses of endothelial cells. RNF213 is a susceptibility gene for moyamoya and it functions to maintain blood flow 
in the case of hypotension in the brain. It is also a genetic risk factor for pulmonary hypertension and systemic vascular disease [21]. RNF213 mutations have been found in many cancers and sarcomas [21], while RNF213 gene translocation occurs in anaplastic large cell lymphoma and inflammatory myofibroblastic tumor [9]. As a member of the SLC26 family, SLC26A11 is mainly involved in anion transportation and acts on homeostasis and intracellular electrolyte balance [9]. The role of SLC26A11 in disease is unclear. Currently, RNF213-SLC26A11 gene fusion has only been reported in chronic myeloid leukemia [9] and glioma [10], which is related to tumor progression. The expression of RNF213-SLC26A11 fusion protein has not been reported previously, and its function in BCOR-CCNB3 sarcomas is unknown. Because RNF213 is involved in angiogenesis and vascular-related lesions, and the BCOR-CCNB3 sarcoma with RNF213-SLC26A11 gene fusion has the thickening of the arterial wall which has not been described for BCOR-CCNB3 sarcomas after neoadjuvant chemotherapy, we speculate that RNF213-SLC26A11 gene fusion may participate in the vascular change of the tumor.

BCOR-CCNB3 sarcomas are relatively rare tumors with no standard therapeutic schedule, but neoadjuvant chemotherapy can effectively improve overall survival (OS) and disease-free interval (DFI) of patients [16]. The commonly used ES chemotherapy regimens extend the OS to $75 \%$ in 5 years $[4,16]$. Some authors believe that the use of clear cell sarcoma of the kidney (CCSK)-based therapy regimens (which emphasize Doxorubicin and do not include ifosfamide) can benefit patients while reducing the toxicity of drugs from ES chemotherapy regimens (which include both doxorubicin and ifosfamide) [22]. In this case, the neoadjuvant chemotherapy with oxaliplatin, pirarubicin and ifosfamide was also effective, resulting in no recurrence or metastasis of the tumor half a year post surgery. Therefore, accumulating cases with different regimens may help to evaluate the chemotherapy protocol of $B C O R-C C N B 3$ sarcomas.

\section{Declarations}

Ethics approval and consent to participate This study was approved by the Institutional Review Board (IRB) of the First Affiliated Hospital of USTC. Consent to participate was acquired.

Consent for publication Written informed consent was obtained from individual or guardian participants.

Data availability Data could be accessed on request from the authors.

Conflict of interest The authors declare no potential conflict of interest.

Funding information This work is supported by the Natural Science Foundation of Anhui Province (N0.1908085MH281).

Authors' contributions WH collected the data and drafted the manuscript. WW conceived the idea and analyzed the data. LH helped perform the analysis with constructive discussions. LH performed the experiment. HW conceived the idea and provided funding acquisition. HW and WZ revised and edited the manuscript. All authors read and approved the final manuscript. 


\section{References}

1. Li WS, Liao IC, Wen MC, Lan HH, Yu SC, Huang HY: BCOR-CCNB3-positive soft tissue sarcoma with round-cell and spindle-cell histology: a series of four cases highlighting the pitfall of mimicking poorly differentiated synovial sarcoma. Histopathology 2016, 69:792-801.

2. Pierron G, Tirode F, Lucchesi C, Reynaud S, Ballet S, Cohen-Gogo S, Perrin V, Coindre JM, Delattre O: A new subtype of bone sarcoma defined by BCOR-CCNB3 gene fusion. Nat Genet 2012, 44:461-466.

3. Miettinen M, Felisiak-Golabek A, Lui?a Contreras A, Glod J, Kaplan RN, Killian JK, Lasota J: New fusion sarcomas: histopathology and clinical significance of selected entities. Hum Pathol 2019, 86:57-65.

4. Kao YC, Owosho AA, Sung YS, Zhang L, Fujisawa Y, Lee JC, Wexler L, Argani P, Swanson D, Dickson BC, et al: BCOR-CCNB3 Fusion Positive Sarcomas: A Clinicopathologic and Molecular Analysis of 36 Cases With Comp arison to Morphologic Spectrum and Clinical Behavior of Other Round Cell Sarcomas. Am J Surg Pathol 2018, 42:604-615.

5. Puls F, Niblett A, Marland G, Gaston CL, Douis H, Mangham DC, Sumathi VP, Kindblom LG: BCORCCNB3 (Ewing-like) sarcoma: a clinicopathologic analysis of 10 cases, in comparison with convent ional Ewing sarcoma. Am J Surg Pathol 2014, 38:1307-1318.

6. Matsuyama A, Shiba E, Umekita Y, Nosaka K, Kamio T, Yanai H, Miyasaka C, Watanabe R, Ito I, Tamaki T, et al: Clinicopathologic Diversity of Undifferentiated Sarcoma With BCOR-CCNB3 Fusion: Analysis of 11 Cases With a Reappraisal of the Utility of Immunohistochemistry for BCOR and CCNB3. Am J Surg Pathol 2017, 41:1713-1721.

7. Ludwig K, Alaggio R, Zin A, Peron M, Guzzardo V, Benini S, Righi A, Gambarotti M: BCOR-CCNB3 Undifferentiated Sarcoma-Does Immunohistochemistry Help in the Identification? Pediatr Dev Pathol 2017, 20:321-329.

8. Peters TL, Kumar V, Polikepahad S, Lin FY, Sarabia SF, Liang Y, Wang WL, Lazar AJ, Doddapaneni H, Chao $\mathrm{H}$, et al: BCOR-CCNB3 fusions are frequent in undifferentiated sarcomas of male children. Mod Pathol 2015, 28:575-586.

9. Zhou JB, Zhang T, Wang BF, Gao HZ, Xu X: Identification of a novel gene fusion RNF213?SLC26A11 in chronic myeloid leukemia by RNA-Seq. Mol Med Rep 2013, 7:591-597.

10. Bao ZS, Chen HM, Yang MY, Zhang CB, Yu K, Ye WL, Hu BQ, Yan W, Zhang W, Akers J, et al: RNA-seq of 272 gliomas revealed a novel, recurrent PTPRZ1-MET fusion transcript in secondary glioblas tomas. Genome Res 2014, 24:1765-1773.

11. Nicorici D, Satalan M, Edgren H, Kangaspeska S, Murumagi A, Kallioniemi O, Virtanen S, Kilkku O: FusionCatcher - a tool for finding somatic fusion genes in paired-end RNA-sequencing data. Biorxiv 2014.

12. Astolfi A, Fiore M, Melchionda F, Indio V, Bertuccio SN, Pession A: BCOR involvement in cancer. Epigenomics 2019, 11:835-855. 
13. Yoshida A, Arai Y, Hama N, Chikuta H, Bando Y, Nakano S, Kobayashi E, Shibahara J, Fukuhara H, Komiyama $\mathrm{M}$, et al: Expanding the clinicopathologic and molecular spectrum of BCOR-associated sarcomas in adults. Histopathology 2020, 76:509-520.

14. Alfaro-Cervello C, Andrade-Gamarra V, Nieto G, Navarro L, Mart"“an-Va?" $® S$, Garc"a a de la Torre JP, Bengoa Caama?o M, Garc"a a Mauri?o ML, Noguera R, Navarro S: Congenital undifferentiated sarcoma associated to BCOR-CCNB3 gene fusion. Pathol Res Pract 2017, 213:1435-1439.

15. Mantilla JG, Ricciotti RW, Chen E, Hoch BL, Liu YJ: Detecting disease-defining gene fusions in unclassified round cell sarcomas using anchored multiplex PCR/targeted RNA next-generation sequencing-Molecular and clinicopathological characterization of 16 cases. Genes Chromosomes Cancer 2019, 58:713-722.

16. Cohen-Gogo S, Cellier C, Coindre JM, Mosseri V, Pierron G, Guillemet C, Italiano A, Brugi' '.'res L, Orbach D, Laurence V, et al: Ewing-like sarcomas with BCOR-CCNB3 fusion transcript: a clinical, radiological and pathological retr ospective study from the Soci"|t|"| Fran?aise des Cancers de L'Enfant. Pediatr Blood Cancer 2014, 61:2191-2198.

17. Yamada Y, Kuda M, Kohashi K, Yamamoto H, Takemoto J, Ishii T, lura K, Maekawa A, Bekki H, Ito T, et al: Histological and immunohistochemical characteristics of undifferentiated small round cell sarcomas as sociated with CIC-DUX4 and BCOR-CCNB3 fusion genes. Virchows Arch 2017, 470:373-380.

18. Shibayama T, Okamoto T, Nakashima Y, Kato T, Sakurai T, Minamiguchi S, Kataoka TR, Shibuya S, Yoshizawa A, Toguchida J, Haga H: Screening of BCOR-CCNB3 sarcoma using immunohistochemistry for CCNB3: A clinicopathological report of three pediatric cases. Pathol Int 2015, 65:410-414.

19. Hwang HC, Mills SE, Patterson K, Gown AM: Expression of androgen receptors in nasopharyngeal angiofibroma: an immunohistochemical study of $24 \mathrm{c}$ ases. Mod Pathol 1998, 11:1122-1126.

20. Fiorelli A, Ricciardi C, Pannone G, Santoro A, Bufo P, Santini M, Serpico R, Rullo R, Pierantoni GM, Di Domenico M: Interplay between steroid receptors and neoplastic progression in sarcoma tumors. $J$ Cell Physiol 2011, 226:2997-3003.

21. Wang $X, Y e M$, Wu $M$, Fang $H$, Xiao $B$, Xie L, Zhu X: RNF213 suppresses carcinogenesis in glioblastoma by affecting MAPK/JNK signaling pathway. Clin Trans/ Onco/2020, 22:1506-1516.

22. Argani P, Kao YC, Zhang L, Bacchi C, Matoso A, Alaggio R, Epstein Jl, Antonescu CR: Primary Renal Sarcomas With BCOR-CCNB3 Gene Fusion: A Report of 2 Cases Showing Histologic Overlap Wi th Clear Cell Sarcoma of Kidney, Suggesting Further Link Between BCOR-related Sarcomas of the Kidney and Soft Tissues. Am J Surg Pathol 2017, 41:1702-1712.

\section{Tables}

Table 1 The histological and immunohistochemocal changes before and after chemotherapy 


\begin{tabular}{|llll|}
\hline & pre-chemotherapy & post-chemotherapy \\
IHC & & small round cells & larger myoid cell \\
\cline { 2 - 3 } BCOR & + & - & + \\
Bcl2 & + & - & + \\
AR & + & - & + \\
SMA & - & - & + \\
SATB2 & Scatter + & - & + \\
CD117 & - & - & + \\
CD56 & Scatter + & - & + \\
CD99 & Scatter + & - & + \\
\hline CyclinD1 & + & - & + \\
\hline TLE1 & + & - & + \\
\hline
\end{tabular}

IHC: Immunohistochemistry; BCOR: BCL6 corepressor; AR: Androgen Receptor; SMA: Smooth muscle Actin; SATB2: Special AT-rich sequence-binding protein 2; TLE1: Transducin-like enhancer protein 1

\section{Figures}



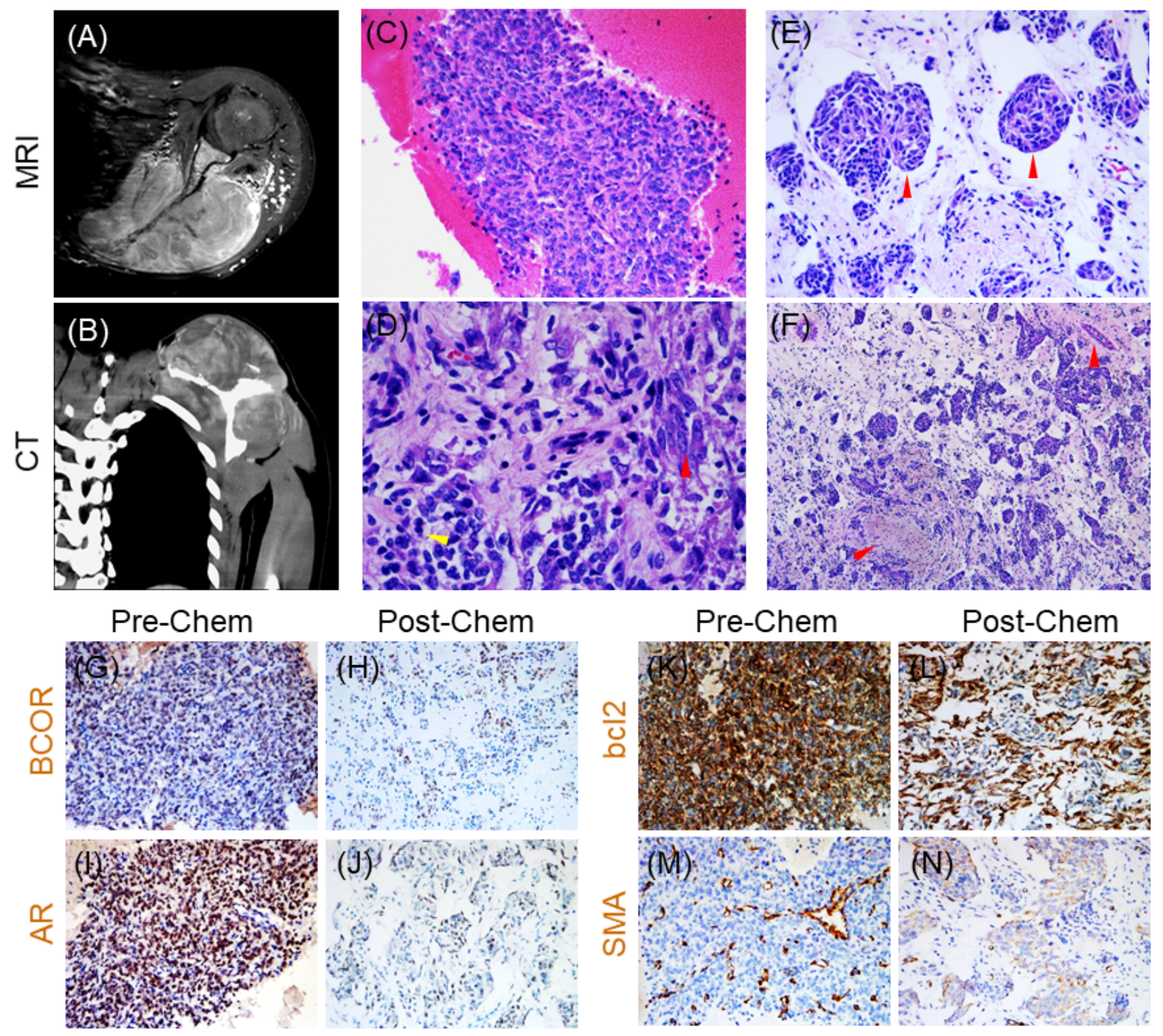

\section{Figure 1}

Histomorphological features of the tumor in this study. (A) The MRI imaging showed a huge soft tissue mass growing around the left scapula. (B) The contrast-enhanced CT showed the tumor grew inside and outside the shoulder blade, and the scapular spine. (C) The pre-chemotherapy biopsy showed spindle and ovoid cells that were arranged in solid sheets. (D) The post-chemotherapy tumor showed the primitive small round cells (yellow arrow) and larger myoid like cells (red arrow). (E) Crescent-shaped gaps were 
noted around the nests, forming a glomerular-like structure in the post-chemotherapy tumor. (F) Vascular stenosis and occlusion were detected in the post-chemotherapy tumor. (G-L) IHC staining demonstrated that BCOR, AR, and bcl2 showed diffuse and strong staining in the pre-chemotherapy biopsy $(\mathrm{G}, \mathrm{l}, \mathrm{K})$, but focal positive mainly in myoid cells in the post-chemotherapy tumor $(\mathrm{H}, \mathrm{J}, \mathrm{L})$. (M and N) IHC showed that SMA expression was negative in most tumor cells in the pre-chemotherapy biopsy ( $M$ ) but positive in myoid cells in the post-chemotherapy tumor $(\mathrm{N})$.

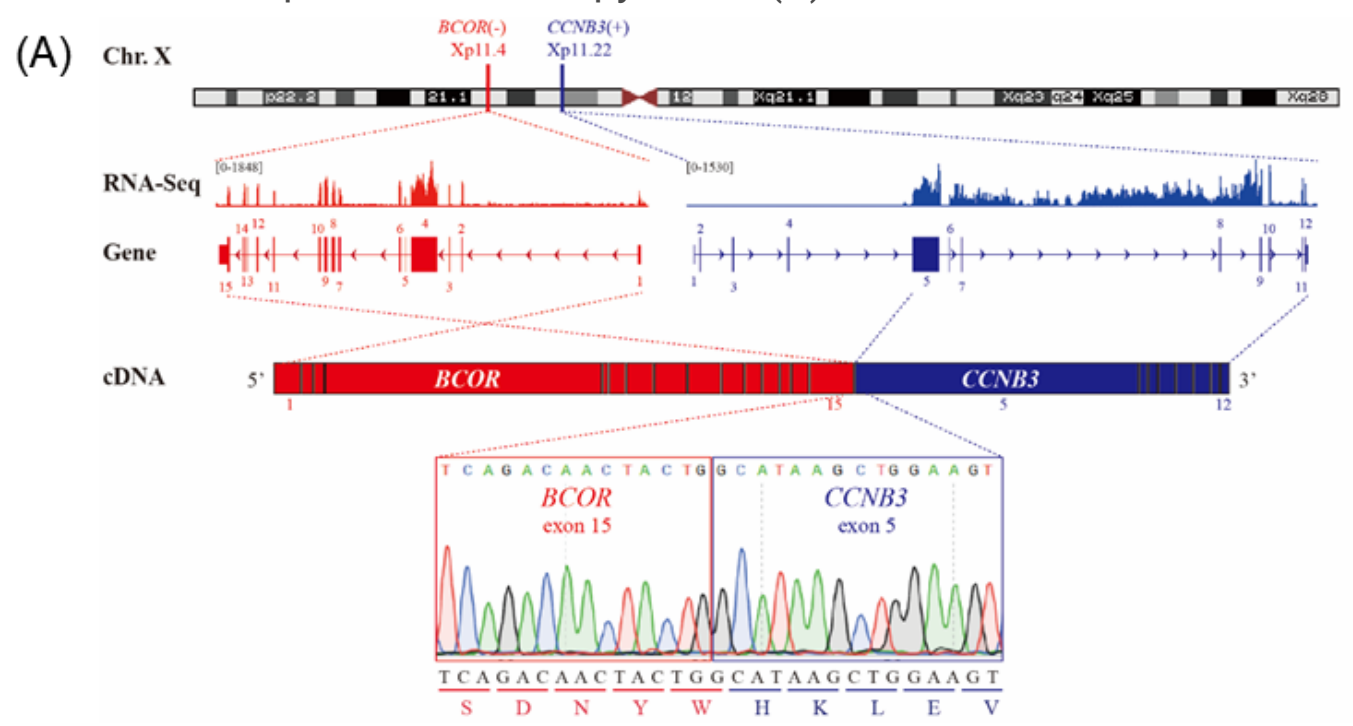

Huang et al. Fig. 2

(B)

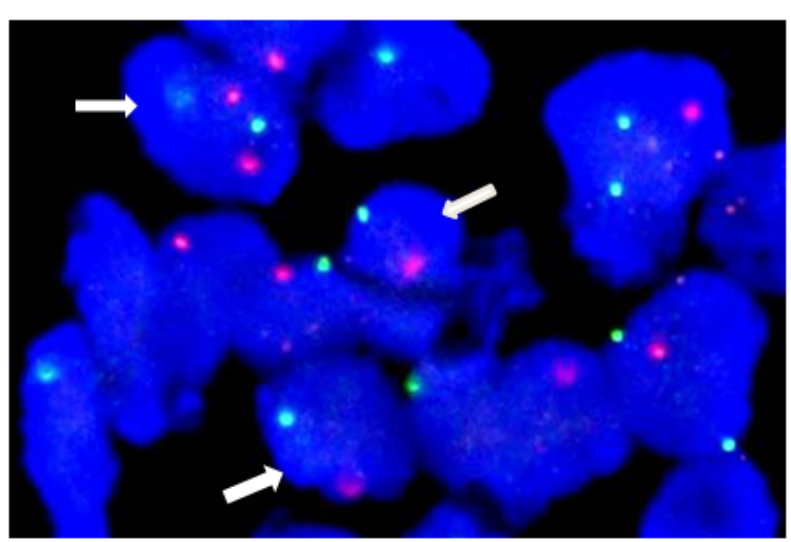

Red: BCOR 5' probe Green: BCOR 3'probe

(C)

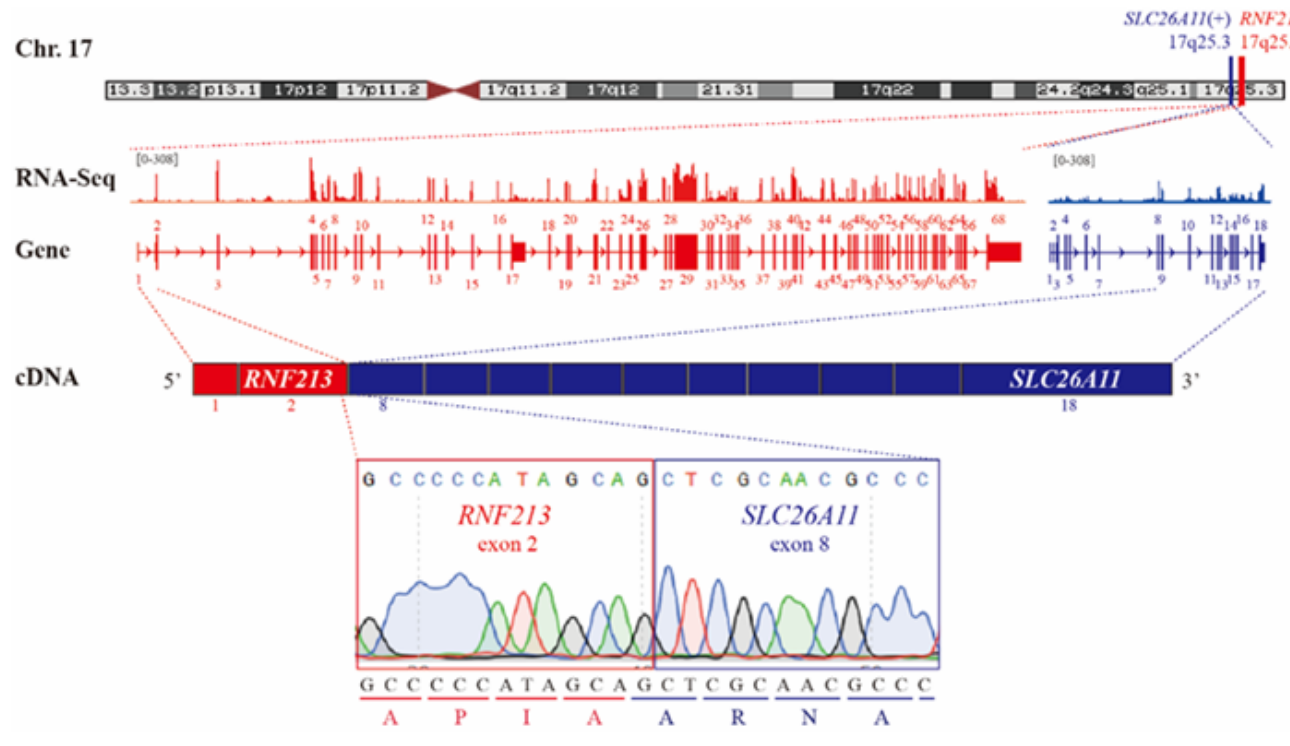

Figure 2 
RNF213-SLC26A11 in addition to BCOR-CCNB3 fusion in the tumor in this study. (A) Schematic diagram (RNA sequencing and Sanger sequence of RT-PCR) showed a gene fusion connecting BCOR exon 15 to CCNB3 exon 5 in this tumor. (B) Fluorescent in situ hybridization study using BCOR break-apart probe demonstrated split of the red ( $\left.5^{\prime}\right)$ and green ( $\left.3^{\prime}\right)$ probes (arrows). (C) RNA sequencing and Sanger sequence of RT-PCR revealed a novel RNF213-SLC26A11 fusion connecting RNF213 exon 2 to SLC26A11 exon 8 in this BCOR-CCNB3 sarcoma. 